\title{
双取代苯的亲电取代反应区域选择性规则的理论解释和实验依据
}

\author{
王佳怡, 许家喜* \\ 北京化工大学化学学院, 有机化学系, 北京 100029
}

摘要: 芳香化合物的亲电取代反应是合成不同取代芳香化合物的重要方法, 芳香亲电取代反应的区域选择性规则是 实现控制合成所需目标分子的理论依据。本文从中间体稳定性、反应活化能和相对反应速率的角度讨论了双取代苯 的芳香亲电取代反应区域选择性规则的理论解释和实验依据, 为学生正确理解该选择性规则提供了合理的解释和实 验证据。

关键词：芳香亲电取代；区域选择性；双取代苯；活化能；相对速率 中图分类号: G64; O624

\section{Theoretical Rationale and Experimental Evidences on Regioselective Rule in Aromatic Electrophilic Substitution of Disubstituted Benzenes}

\author{
Jiayi Wang, Jiaxi $\mathrm{Xu}^{*}$
}

Department of Organic Chemistry, College of Chemistry, Beijing University of Chemical Technology, Beijing 100029, China.

\begin{abstract}
Aromatic electrophilic substitution is a very important method to synthesize different substituted arenes. Its regioselective rule is the theoretical evidence to realize the controlled synthesis of the desired target molecules. Theoretical rationale and experimental evidences on the regioselective rule in the aromatic electrophilic substitution of disubstituted benzenes are discussed here from the viewpoints of stabilities of intermediates, activation energies, and relative reaction rates. It would provide reasonable explanation and experimental evidence for the regioselective rule and is convenient for students to understand correctly.
\end{abstract}

Key Words: Aromatic electrophilic substitution; Regioselectivity; Disubstituted benzene; Activation energy; Relative rate

芳香化合物的亲电取代反应是合成不同取代芳香化合物的重要方法，芳香亲电取代反应的区域 选择性规则是实现控制合成所需目标分子的理论依据。双取代苯的芳香亲电取代反应的区域选择性 规则虽然在各种有机化学教科书中有不同的描述 ${ }^{[1-6]}$, 但概括起来可以总结为：1) 两个取代基作用 位点一致时, 共同决定, 但位阻小的位置有利。2) 两个取代基作用位点不一致时, (a) 致活取代基 的作用大于致钝取代基, 致钝的邻对位定位取代基卤素的定位作用大于致钝的间位定位取代基; (b) 电 子效应和定位效应相同的同类取代基, 电子效应强取代基的作用大于电子效应弱取代基; (c) 两个电 子效应强度差别不大的同类取代基, 得到混合物; 有多个反应位点时, 空间位阻小的有利。由于很 少有教科书详细解释这个选择性规则的原因 ${ }^{[1,2]}$, 造成很多学生学习时只是背下了规则, 没有明白其

收稿: 2020-07-07; 录用: 2020-08-19; 网络发表: 2020-09-15

“通讯作者, Email: jxxu@mail.buct.edu.cn

基金资助: 《有机化学》和《中级有机化学》一流课程建设项目 
中的道理。甚至还会产生一些困惑, 如, 为什么弱给电子取代基的定位作用比强吸电子取代基还强? 给电子取代基的定位作用大于吸电子取代基, 并且强给电子取代基的作用大于弱给电子取代基, 那 为什么强吸电子间位定位取代基的定位作用要比弱吸电子间位定位取代基的强? 因此, 我们认为有 必要从中间体稳定性、反应活化能和反应相对速率的观点来讨论一下这个问题, 希望本文能够对教 师授课和学生学习和理解芳香亲电取代反应的区域选择性规则提供有益的帮助。

\section{1 不同取代基对芳香亲电取代反应活化能和相对反应速率的影响}

苯环上的取代基分为致活基团和致钝基团两大类。致活基团为给电子取代基，可以增加苯环的 电子密度, 有利于苯环发生亲电取代反应。尤其是致活基团可以稳定在苯环上邻对位反应时形成的 环己二烯正离子中间体。另外, 对于弱致钝的卤素类取代基, 由于在其邻对位反应时, 当正电荷在 与卤素相连的碳原子上时, 卤素与其共轭可以形成具有八电子结构的稳定共振式, 也可以稳定在苯 环上邻对位反应时形成的环己二烯正离子中间体。因此, 有利于在其邻对位反应, 致活基团和弱致 针的卤素就是邻对位定位基。致针基团是吸电子取代基, 会降低苯环的电子密度, 因此, 不利于苯 环发生亲电取代反应。除了卤素以外, 含有吸电子取代基的苯如果在其邻对位发生亲电取代反应就 会形成特别不稳定的环已二烯正离子中间体，因此，其亲电取代反应只能发生在取代基的间位 ${ }^{[1,2]}$ 。 芳香亲电取代反应的第一步是速控步, 且是后过渡态, 过渡态的结构应该与环己二烯正离子中间体 的结构接近。根据以上结果和 Hammond 假说, 中间体稳定的反应过程的过渡态能量也应该低。因 此, 不同类型取代苯在邻对位和间位发生亲电取代反应进程的活化能次序应该为:

$E_{\mathrm{a}}$ (致活基团邻对位 $)<E_{\mathrm{a}}$ (致活基团间位 $)<E_{\mathrm{a}}$ (苯) $<E_{\mathrm{a}}$ (卤素邻对位 $)<E_{\mathrm{a}}$ (卤素间位 $)<E_{\mathrm{a}}$ (致钝 基团间位) $<E_{\mathrm{a}}$ (致针基团邻对位)。

根据阿仑尼乌斯公式: $k=A \mathrm{e}^{\left(-E_{\mathrm{a}} / R T\right)}, k$ 为反应速率常数, $A$ 为指前因子, $E_{\mathrm{a}}$ 为活化能, $R$ 为比例 系数, 也称为摩尔气体常数, $T$ 为反应温度(开氏温度)。在相同反应条件下, 由于是同一类型的相似 反应, $A$ 可以认为差别不大。因此, $E_{\mathrm{a}}$ 越小, 反应速率常数就越大, 反应速率也就越大。

由此可见, 在相同反应条件下, 苯环上亲电取代反应速率的大小顺序应该为: $v$ (致活基团邻对 位 $)>v$ (致活基团间位 $)>v$ (苯) $>v$ (卤素邻对位 $)>v$ (卤素间位 $)>v$ (致钝基团间位 $)>v$ (致针基团邻 对位)。这就是取代基定位效应产生的原因。虽然很多教材会给出这些类型亲电取代反应的相对反应 速率, 但是学生不容易记住。通过这样的分析, 便于学生理解, 也可以把绪论中学习的知识再巩固 一下, 体会知识的连贯性和系统性, 学会知识的综合应用。

\section{2 双取代苯芳香亲电取代反应区域选择性规则的解释和实验证据}

对于区域选择性规则 1 的 “两个取代基作用位点一致时, 共同决定”, 和 2c “两个电子效应强度 差别不大的同类取代基, 得到混合物”, 这两条不需要解释。下面我们重点解释一下 $2 \mathrm{a}$ 和 $2 \mathrm{~b}$ 这两条。

\section{1 活化取代基的作用大于针化取代基}

既然致活基团(electron-donating group, EDG)可以增加苯环的电子密度, 致钝基团(electronwithdrawing group, EWG)可以降低苯环的电子密度, 那为什么双取代苯芳香亲电取代反应的区域选 择性由致活基团决定呢? 我们先来看同时含有致活和致针基团的双取代苯的亲电取代反应中间 体, 由于卤素是吸电子的邻对位定位基, 后面要讨论, 这里讨论了三种不同取代位置的异构体。从 下列发生亲电取代反应第一步亲电加成的中间体可以看出, 不论致针基团在致活基团的邻间对哪个 位置, 亲电试剂 $\left(\mathrm{E}^{+}\right.$)在致活基团的邻位加成时都能够形成稳定的中间体(此处只画出了中间体最稳定 的共振极限式, 省略了中间体的其他共振极限式, 因为中间体的共振杂化体主要就是由最稳定的共 振极限式决定的)。除了当致针基团在致活基团的对位时, 亲电试剂在致活基团的对位加成时, 也能 够形成稳定的中间体。而在间位加成时都不能形成稳定的中间体, 甚至还可以形成特别不稳定的中 间体。因此，致活基团就决定了双取代苯芳香亲电取代反应的区域选择性(图 1)。 
在该类芳香亲电取代反应中, 大多数例子学生都容易理解。但是, 部分学生对对卤甲苯类型底 物亲电取代反应的区域选择性比较困惑, 因为在卤素的邻位反应时, 形成的中间体可以具有八电子 结构的稳定共振式。而在甲基的邻位反应时, 形成的中间体只是具有三级碳正离子(图 2)。到底哪个 中间体更稳定? 到底是有利于在甲基还是卤素的邻位发生亲电取代反应? 让我们以对氯甲苯为例来 看看实验事实。其氯化反应中甲基邻位氯代产物占有绝对优势 ${ }^{[7]}$, 溴化 ${ }^{[8]}$ 和酰化 ${ }^{[9]}$ 反应只给出了甲基 邻位发生反应的产物。说明均有利于在甲基的邻位发生亲电取代反应, 支持致活基团甲基控制反 应的区域选择性, 应该是甲基的给电子作用对于稳定碳正离子的作用比弱吸电子的卤素的作用大 (图 2)。

对于同时含有邻对位定位的弱致钝基卤素和间位致针基的二取代苯，由于在卤素的邻对位反应 形成的中间体都可以形成具有八电子结构的稳定共振式, 而有利于在卤素的邻对位发生亲电取代反 应(图 3)。

\section{2 电子效应和定位效应相同的同类取代基，电子效应强取代基的作用大于电子效应弱取代基}

强致活基团的影响比弱致活基团的大, 这一点很容易理解。因为强致活基团对在其邻对位发生 亲电取代反应形成的中间体中, 有正电荷在强致活基团连接的碳原子上的共振式, 稳定作用强, 所 以, 强致活基团的影响比弱致活基团的大。

强间位定位钝化基团(strong electron-withdrawing groups, EWG)的定位作用大于弱间位定位钝化 基团(weak electron-withdrawing group, wEWG)。关于强间位定位针化基团的定位作用大于弱间位定 位钝化基团有些学生不易理解。他们认为弱间位定位钝化基团的吸电子能力相对较弱, 从某种意义 上讲, 其钝化苯环的作用就会比强钝化基团的弱, 似乎应该由其决定亲电取代反应的区域选择性。

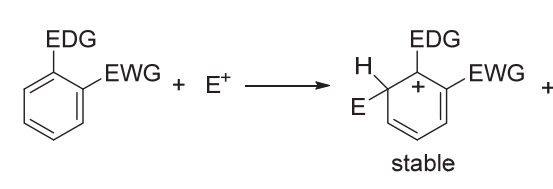<smiles>CC(C)(C)c1cccc(C(=O)OCc2cccc(C(=O)OCc3cccc(C(=O)OCc4ccccc4)c3)c2)c1</smiles>

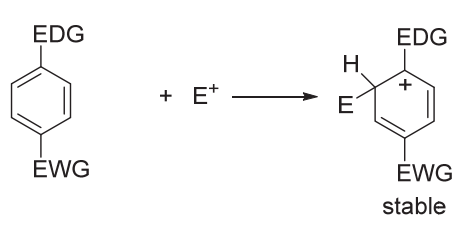
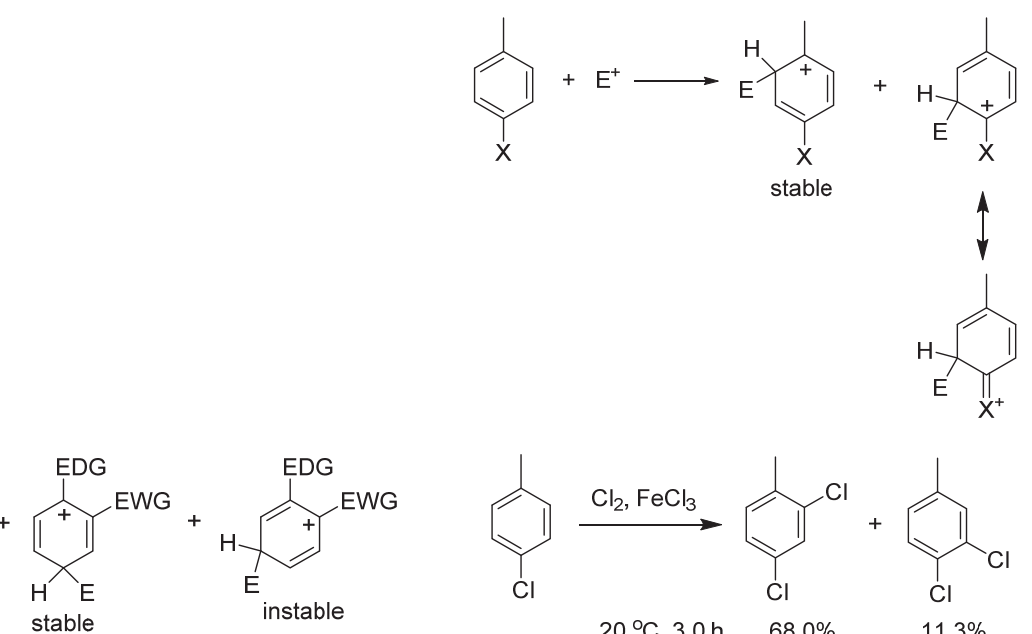

EDG<smiles>CC(C)(C)c1cc(C(=O)O[Na])ccc1[18O]</smiles><smiles>COC(=O)c1cc(F)cc(C(=O)O[Na])c1</smiles><smiles>O=[W]c1ccc(C(=O)OCc2ccccc2)cc1</smiles>

图 1 同时含有致活和致针基团的双取代苯的亲电取代反应

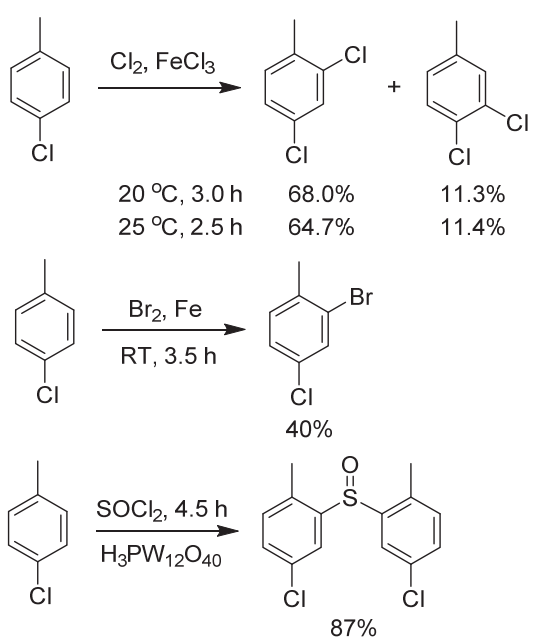

图 2 对卤代甲苯的亲电取代反应 

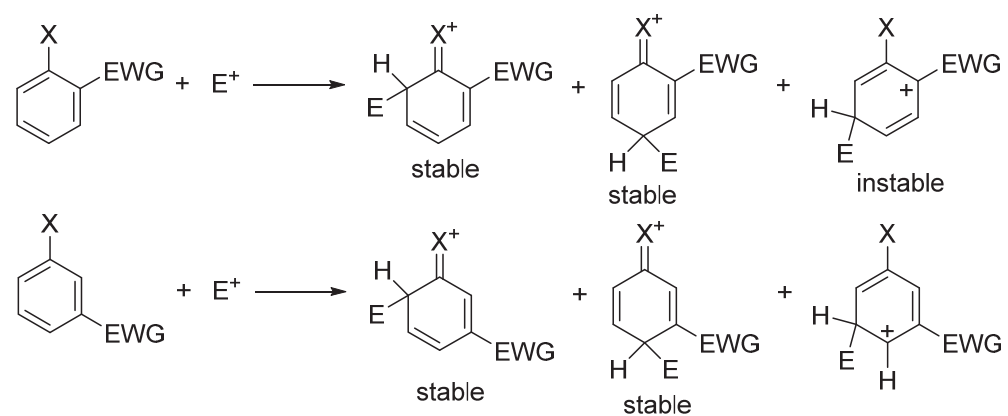<smiles>[X]c1ccc(C(C)(C)C)cc1</smiles>

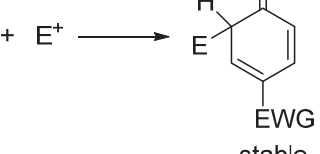<smiles>[X]c1ccc([R16]([H])([H])[H])c(F)c1</smiles>

图 3 同时含有邻对位定位弱致针和间位定位致钝基团的双取代苯的亲电取代反应

我们首先来看看这两个基团处于邻位和对位时, 发生亲电取代反应形成中间体的稳定性。当两 个基团处于邻位时，亲电试剂进攻强致钝基团的间位时形成的中间体的稳定性比进攻强钝化基团的 邻对位时形成的中间体要稳定(由于进攻不同位置形成的中间体共振式的数目是一样的, 这里只画出 了不同中间体中最不稳定的共振式), 因为其最不稳定的共振式是正电荷在连有弱致钝基团的碳原子 上。当两个基团处于对位时, 亲电试剂进攻强致钝基团的间位时形成的中间体的稳定性比进攻强钝 化基团的邻位时形成的中间体要稳定(同上, 也只画出了中间体中最不稳定的共振式)。根据中间体 的稳定性，强间位定位钝化基团的定位作用大于弱间位定位钝化基团(图 4)。

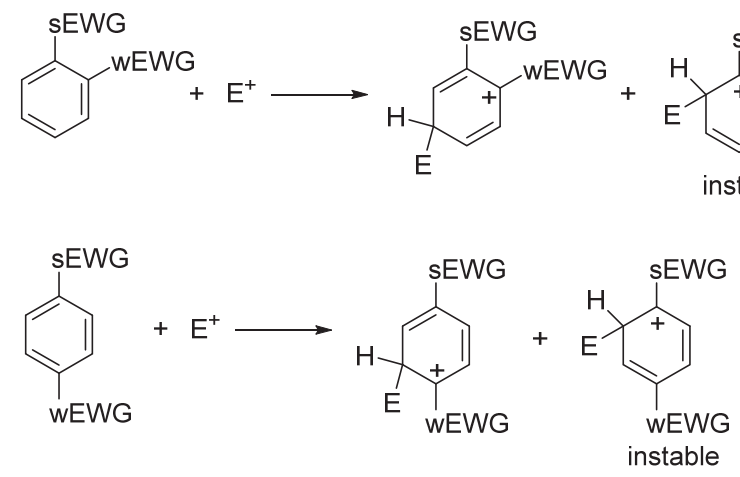

\section{图 4 含有双间位定位致钝基团双取代苯的亲电取代反应}

有学生提出, 中间体的共振杂化体主要是由稳定的共振式的结构决定的, 最不稳定的共振极限 式对中间体的稳定性应该影响比较小。这样分析合理吗? 尽管几本教材都是这样描述的, 强钝化基 团的定位作用大于弱针化基团, 考虑到很多教材的编写有趋同效应。让我们来看看实验事实, 首先 查阅了教材给出的底物对硝基苯甲酰胺 ${ }^{[4]}$, 对硝基苯甲酸 ${ }^{[5]}$, 邻/对氧基苯甲酸 ${ }^{[6]}$ 的常见亲电取代反 应, 并没有找到实例。双吸电子基取代的苯由于电子密度很低, 较难发生亲电取代反应, 该类底物 文献报道确实很少, 仅发现两例。其中, 邻硝基苯甲酫的溴化研究得非常仔细, 为这类底物的亲电 取代反应区域选择性提供了实验支持 ${ }^{[10]}$ 。结果表明, 由于硝基的吸电子作用比酰基强, 溴化主要发 生在硝基的间位。4-硝基苯乙酮的溴化也是发生在硝基的间位 ${ }^{[11]}$ 。实验结果与对中间体的稳定性的 分析结果是一致的。表明虽然中间体的共振杂化体主要是由稳定的共振式的结构决定的, 但最不稳 定的共振极限式对中间体的稳定性还是产生了明显的影响(图 5)。 


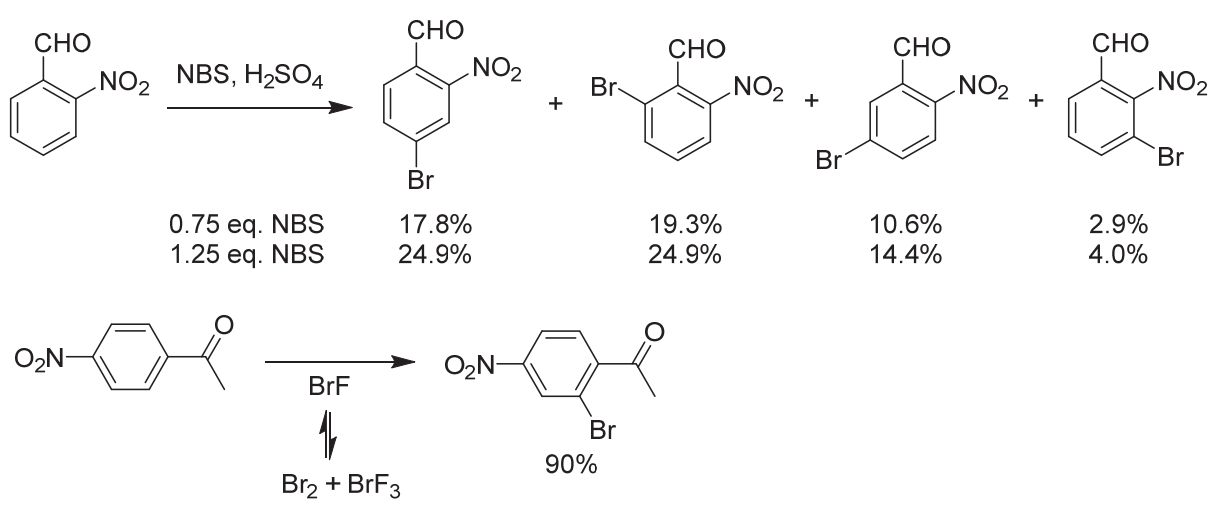

图 5 含有双间位定位致针基团双取代苯的亲电取代反应实例

我们以上讨论的双取代苯发生芳香亲电取代反应的区域选择性是在没有其他因素影响下的区域 选择性, 有多个反应位点时, 亲电试剂一般有利于进攻位阻小的反应位点。但是, 当亲电试剂与苯 上取代基有特殊作用时，如形成氢键或者有静电相互作用，将有利于在其邻位发生反应。

\section{3 结语}

本文结合芳香亲电取代反应中第一步亲电加成中间体的稳定性、Hammond 假说、过渡态能量、 反应活化能和相对反应速率, 以及文献报道的实例来解释说明双取代苯的亲电取代反应区域选择性 规则的理论解释和实验依据, 讨论了双取代苯的亲电取代反应区域选择性的规则。我们一直致力于 对有机化学的教学内容开展研究 ${ }^{[12-15]}$, 希望能够对教师授课和学生的学习和理解提供有益的帮助。

\section{参 考 文 献}

[1] Volhardt, K. P. C.; Schore, N. E. Organic Chemistry, 8th ed.; McMilan Education: New York, USA, 2018 ; pp. $773-775$.

[2] 邢其毅, 裴伟伟, 徐瑞秋, 裴坚. 基础有机化学. 第 4 版. 北京: 北京大学出版社, 2017: 780-782.

[3] 胡宏纹. 有机反应. 第 4 版. 北京: 高等教育出版社, 2013: 208-209.

[4] 李艳梅, 赵圣印, 王兰英, 罗自萍, 李兆陇, 黄智敏, 麻远. 有机化学. 第 2 版. 北京: 科学出版社, 2016: 98-99.

[5] 高占先. 有机反应. 第 3 版. 北京: 高等教育出版社, 2018: 206-207.

[6] 李小瑞, 姚团利, 赵艳娜, 张金. 有机反应. 第 2 版. 北京: 化学工业出版社, 2018: 167-168.

[7] 陈钟秀, 王刚, 赖月琴. 化学工程, 2001, 29 (5), 66.

[8] Campiani, G.; Butini, S.; Fattorusso, C.; Trotta, F.; Gemma, S.; Catalanotti, B.; Nacci, V.; Fiorini, I.; Cagnotto, A.; Mereghetti, I.; et al. J. Med.. Chem. 2005, 48, 1705.

[9] Bhilare, S. V.; Deorukhkar, A. R.; Darvatkar, N. B.; Rasalkar, M. S.; Salunkhe, M. M. J. Mol. Catal A: Chem. 2007, $270,123$.

[10] Söderberg, B. C. G.; Cummings, M. M. Synth. Commun. 2014, 44, 954.

[11] Rosen, S.; Lerman, O. J. Org. Chem. 1993, 58, 239.

[12] 许家喜. 大学化学, 2013, 28 (3), 77.

[13] 许家喜. 大学化学, 2013, 28 (5), 349.

[14] 许薇, 许家喜. 大学化学, 2016, 31 (8), 60.

[15] 许家喜, 陈宁. 大学化学, 2020, 35 (7), 166. 